\title{
Gastric superficial neoplasia: high miss rate but slow progression
}

\section{(ㄷ)(1) $\odot$}

\author{
Authors \\ Yuichi Shimodate, Motowo Mizuno, Akira Doi, Naoyuki Nishimura, Hirokazu Mouri, Kazuhiro Matsueda, Hiroshi \\ Yamamoto
}

Institution

Department of Gastroenterology and Hepatology, Kurashiki Central Hospital, 1-1-1, Miwa, Kurashiki, Okayama, Japan

submitted 20.6.2016

accepted after revision 2.3.2017

Bibliography

DOI https://doi.org/10.1055/s-0043-110076 |

Endoscopy International Open 2017; 05: E722-E726

(c) Georg Thieme Verlag KG Stuttgart · New York

ISSN 2364-3722

Corresponding author

Yuichi Shimodate, MD, Department of Gastroenterology and Hepatology, Kurashiki Central Hospital, 1-1-1 Miwa, Kurashiki, Okayama 710-8602, Japan

Fax: +81-86-431-3424

ys13544@kchnet.or.jp

\section{ABSTRACT}

Background and aims Gastric superficial neoplasia (GSN) is often overlooked at endoscopy because of difficulty in identifying it. The miss rate of GSN at endoscopy and the impact on clinical outcome of the missed GSN have not been fully elucidated. In this study, we investigated these issues.
Methods Among 1462 endoscopically and pathologically diagnosed gastric cancers in our hospital from January 2011 to December 2014, previous records of esophagogastroduodenoscopy (EGD) were available for 198 lesions (index lesions) and were reviewed retrospectively. Among those, 157 lesions, which were diagnosed as GSN on the basis of their EGD findings at initial endoscopy, were analyzed. Progression was defined as advanced cancer in the index lesion.

Results Among the 157 GSNs, 118 (75.2\%) had not been recorded in the previous EGD report but were evident upon review of endoscopic photographs for this study. Progression to advanced cancer was observed in only 13 (8.3\%) of the 157 GSNs during a mean interval of 41 months and as long as 96 months, and the rate of progression was similar in missed and not-missed lesions ( $8.5 \%$ and $7.7 \%$, respectively). Cumulative incidence rates of progression of missed GSNs to advanced cancer were $0.8 \%, 1.7 \%, 4.2 \%$, and $7.6 \%$ at $36,48,60$, and 72 months after the initial EGD, respectively.

Conclusions Our findings illustrate that GSNs are often missed at endoscopy but progress slowly in most cases. Even though the rate of progression to cancer is relatively low, rigorous attempts should be made to reduce the miss rate of GSNs at EGD.

\section{Introduction}

In parallel with recent improvements in esophagogastroduodenoscopy (EGD) such as magnifying endoscopy with narrowband imaging, the diagnostic accuracy of EGD is improving and the detection rate of early gastric cancers and precancerous lesions is increasing. Also, endoscopic submucosal dissection has become a widely accepted procedure for the treatment of gastric superficial neoplasia (GSN) [1].

GSN is often easily overlooked endoscopically because of the difficulty in recognizing it. Several retrospective studies have investigated the miss rate of upper gastrointestinal cancers at endoscopy [2-4], in which missed cancer was defined as cancer detected within 3 years after preceding EGD. However, no report has focused on miss rates of GSN, and the impact on the clinical outcome of missed GSNs has not been clarified.

In this study, we retrospectively investigated the natural history and clinicopathological features of missed GSNs by reviewing patients' preceding endoscopic records and the patients' subsequent clinical outcomes.

\section{Patients and methods}

\section{Patients}

In this retrospective observational study, 1462 consecutive lesions, which were endoscopically and pathologically diagnosed as gastric cancer in Kurashiki Central Hospital from January 2011 to December 2014, were evaluated. Among the 1462 le- 
sions, EGD had been performed in our hospital after January 2006 and at least 6 months before the index EGD in 198 lesions (index lesions). Five gastrointestinal endoscopists (Y.S., M. M., A.D., N.N., and H.M, who are board-certificated members of the association of Japan Gastroenterological Endoscopy Society, separately reviewed all records of these 198 lesions. We excluded the following lesions: (1) lesions without suspicious preceding lesion in the previous EGD records (14 lesions); (2) lesions diagnosed as invasive cancer at the initial EGD (3 lesions); and (3) lesions without $100 \%$ agreement on the findings among all five reviewers (24 lesions). The remaining 157 lesions, which were diagnosed as GSN on the basis of their EGD findings at initial endoscopy, were enrolled ( $\triangleright$ Fig. 1). Rates of inter-observer agreement on the presence of suspicious preceding lesion and the diagnosis of invasive cancer were $87.9 \%$ and $100 \%$, respectively. The mean interval from the initial EGD to the final pathological diagnosis with staging was 41 months, and the maximum interval was 96 months.

\section{Diagnosis of the index gastric cancer}

For patients who had undergone surgical or endoscopic resection of their lesions, the histologic type and depth of invasion were judged by pathological diagnosis of the resected specimens according to the Japanese Classification of Gastric Carcinoma (3 rd English edition) [5]. Pathological diagnosis of intramucosal gastric cancer in our hospital corresponds mostly to category 5-1 in the Vienna classification [6] and occasionally to category 4 . Tumors that had invaded the muscularis propria or deeper were designated advanced gastric cancer.

For patients who had undergone chemotherapy or supportive care, the histological diagnosis was made on the basis of the findings of endoscopic biopsies. The depth of invasion was judged by comprehensive evaluation with diagnostic studies: EGD, computed tomography, and barium meal. The histological diagnosis was classified as intestinal or diffuse type, according to Lauren [7]; when both types were present, the lesion was classified according to the dominant histological type.

\section{Diagnosis of the initial lesion as GSN}

Diagnosis of the initial lesion as GSN was based on (1) endoscopic photographic findings of the EGD records in which plausible lesion was observed at the site of the index lesion, and findings indicating invasive cancer, e.g. thickening or decreased distensibility of the gastric wall or abnormal appearance of folds of the lesion, were absent, and (2) histological findings of gastric biopsies when they were available. As stated earlier in the Patients subsection, we only enrolled GSNs based on the diagnosis with agreement of all five reviewers.

The study was conducted according to the guidelines of the Declaration of Helsinki. The institutional review board of Kurashiki Central Hospital approved the study protocol.

\section{Statistical analysis}

Demographics of GSN were compared using Fisher's exact test and Student's $t$ test. For the statistical analyses, we used EZR (Saitama Medical Center, Jichi Medical University, Saitama, Ja-

\section{2 gastric cancer at index EGD}

$\downarrow$

198 lesions whose endoscopic images were available for reviewing

14 lesions without suspicious preceding lesion in the previous EGD records

24 lesions without $100 \%$ agreement on the findings among all 5 reviewers

\section{0 suspicious preceding lesions in the record of previous EGD \\ 3 lesions diagnosed as invasive cancer at the initial EGD}

157 GSNs likely to be preceding lesions for the index cancer were studied

\begin{tabular}{|c|c|}
\hline$\sqrt{ }$ & $\downarrow$ \\
\hline $\begin{array}{c}118(75.2 \%) \text { were missed } \\
\text { by examiner }\end{array}$ & $\begin{array}{l}39(24.8 \%) \text { were } \\
\text { recognized by examiner }\end{array}$ \\
\hline$\downarrow$ & $\downarrow$ \\
\hline $\begin{array}{c}10(8.5 \%) \text { progressed to } \\
\text { advanced cancer }\end{array}$ & $\begin{array}{l}3(7.7 \%) \text { progressed to } \\
\text { advanced cancer }\end{array}$ \\
\hline
\end{tabular}

- Fig. 1 Flow chart of study design and natural history of GSN. EGD, esophagogastroduodenoscopy; GSN, gastric superficial neoplasia.

pan), which is a graphical user interface for $R$ (The R Foundation for Statistical Computing, Vienna, Austria).

\section{Results}

\section{Miss rate of GSN}

Among the 157 preceding suspicious GSNs, 118 had not been recorded in the EGD report but were evident upon review of the endoscopic photographs for this study. Thus, the miss rate of GSN in the routine clinical setting of EGD at our hospital was $75.2 \%$ ( $\triangleright$ Fig. 1). The other 39 lesions had been endoscopically identified and recorded in the EGD report but were followed without operation or endoscopic submucosal resection. The reasons for non-interventional follow-up of these lesions were: (1) histological diagnosis of the biopsy specimens was low grade adenoma (category 3 in the Vienna classification; 20 lesions) or hyperplastic polyp (3 lesions); (2) neoplasia was suspected endoscopically but was not proven histologically, probably due to inadequate biopsy specimens (12 lesions); (3) lesions were endoscopically regarded as benign without having been biopsied (2 lesions); or (4) lesions were endoscopically and histologically diagnosed as gastric cancer (2 lesions) but patients refused treatment. Endoscopic photographs of a typical case of missed GSN are presented in $>$ Fig. 2.

Clinicopathological features of missed and not-missed GSNs based on the final reports are shown in $>$ Table 1 . Morphology 

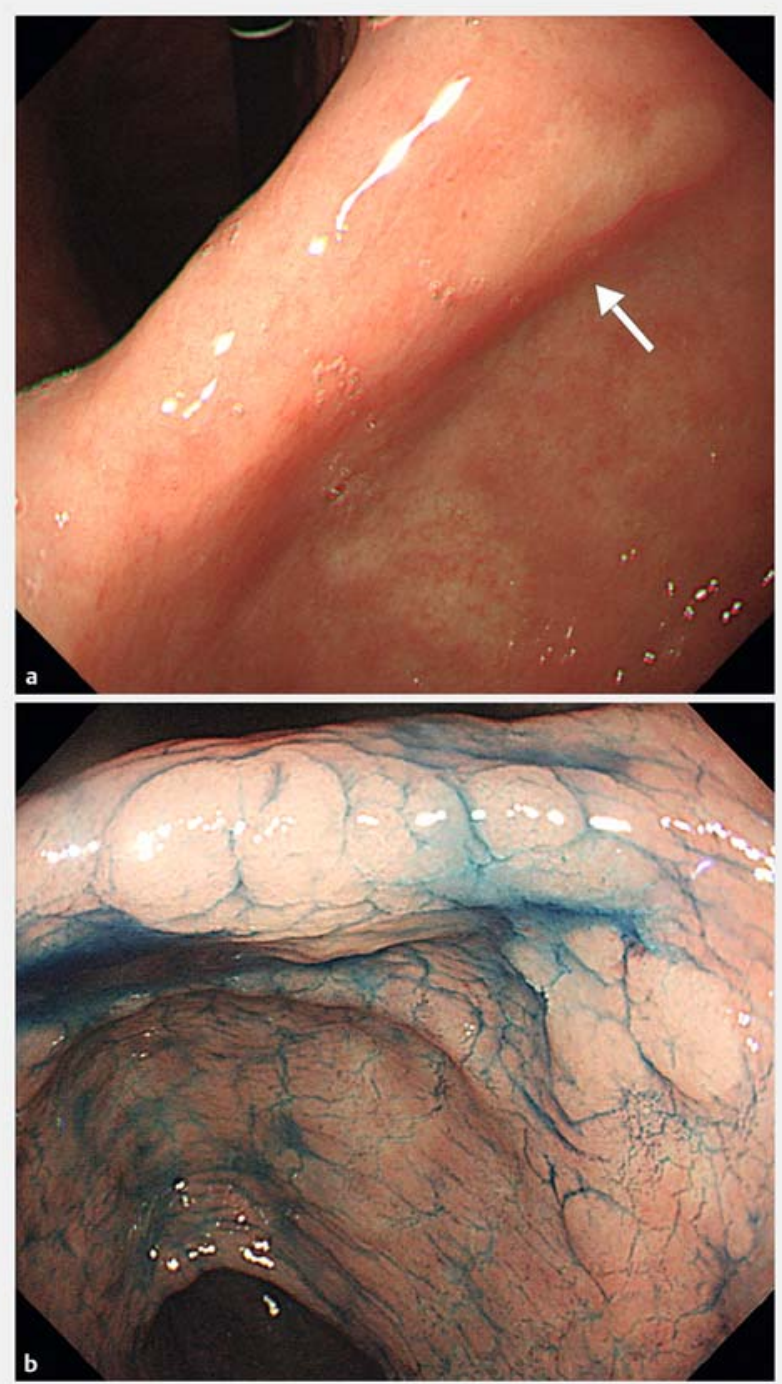

Fig. 2 Endoscopic photographs of a typical case of missed GSN. a A small, pale area is seen on the posterior wall of the angulus (arrow). This lesion was missed at initial endoscopy but was identified at review of photographs for this study. $\mathbf{b}$ Chromoendoscopic imaging of the suspected area with indigo carmine 51 months after the initial endoscopy. The lesion is now larger and somewhat elevated, and was resected by endoscopic submucosal dissection. Histological diagnosis of the resected specimen was intestinal-type, intramucosal gastric cancer.

and histological type of the index lesion were significantly different between the two groups.

\section{Natural history of GSN}

The natural history of the GSNs is illustrated in $>$ Fig. 1. Among the 118 missed GSNs, 10 (8.5\%) progressed to advanced cancer during a mean interval of 39.3 months and as long as 96 months; among the 39 not-missed lesions, 3 (7.7\%) progressed to advanced cancer during a mean interval of 47.5 months and as long as 96 months. Progression rate was statistically insignificant between the two groups. In total, progression to advanced cancer was observed in only 13 (8.3\%) of the 157
GSNs. In the missed GSNs, the cumulative incidence rate of progression to advanced cancer was calculated as $0.8 \%, 1.7 \%$, $4.2 \%$, and $7.6 \%$ at $36,48,60$, and 72 months after the initial EGD, respectively.

\section{Discussion}

In this study, by reviewing the previous EGD records of 198 patients with gastric cancer, we identified 157 GSNs that likely were preceding lesions for the index gastric cancer. Disturbingly, $75.2 \%$ of these GSNs were overlooked at previous EGD. The miss rate of GSN in our study is strikingly higher than miss rates of gastric cancer in published reports, in which rates ranged from $4.6 \%$ to $25.8 \%$ [2-4]. In those studies, missed cancer was defined as cancer that had not been diagnosed at endoscopy performed within 3 years before the diagnosis on the basis of estimated doubling time of mucosal gastric cancer of 2 to 3 years [8]. In our patients, the interval between the previous EGD and EGD at the time of diagnosis of gastric cancer exceeded 3 years to as long as a maximum of 8 years in $57.3 \%(90 / 157)$ (data not shown). The major reason for the higher miss rate in our series compared with other series is probably the fact that we were studying only GSNs, which are more difficult to detect than other gastric neoplasms. It is also possible that observer bias played a role in the high miss rate. That is, the endoscopists who reviewed the initial endoscopic photographs were aware that they were participating in a study of overlooked GSN; thus, they may have looked more zealously for subtle mucosal abnormalities than did the original endoscopists.

In spite of the high miss rate of GSN in our series, we found that only $8.5 \%$ of the overlooked GSNs progressed to advanced cancer during the interval of a mean of 39.3 months and as long as 96 months. This progression rate is much lower than the $33.3 \%$ of missed gastric cancer in the study reported by Hosokawa et al. [9]. Those authors reviewed 250 gastric cancer patients with EGD records available within the previous 3 years, and in whom gastric cancer had not been diagnosed at the previous EGD. The most likely explanation for the difference in progression rates between the two studies is, again, because we only studied GSNs, which probably have less malignant potential than do other gastric neoplasms. In other reports on the natural progression of gastric neoplasms [10-15], Park et al. [11] reviewed records of 26 lesions of gastric adenomas and observed progression of histological grade in 8 (29.6\%) during follow-up of a median of 66 months. Yamada et al. [12] followed 48 gastric adenoma/dysplasia lesions for a median of 4.7 years and found that 1 of 38 (2.6\%) low grade adenoma/dysplasia and 1 of 10 (10\%) high grade adenoma/dysplasia progressed to noninvasive carcinoma and intramucosal carcinoma. Thus, most adenomatous lesions progress quite slowly. We assume that the majority of GSNs in our study were adenomatous or premalignant lesions, and these lesions also progressed slowly.

We found that the cumulative incidence rate of progression of GSNs to advanced cancer was very low (0.8\%) until 36 months after the initial EGD even when they were overlooked. However, the rate increased rapidly afterwards. Thus, we should perform surveillance EGD at least every 36 months to 
- Table 1 Clinicopathological features of missed and not-missed GSNs based on finding in the final reports of index lesions.

\begin{tabular}{|c|c|c|c|}
\hline & $\begin{array}{l}\text { Missed GSNs } \\
(n=118)\end{array}$ & $\begin{array}{l}\text { Not-missed GSNs } \\
(\mathrm{n}=39)\end{array}$ & $P$ value \\
\hline Age, mean $\pm S D$, years & $71 \pm 9.3$ & $72 \pm 9.6$ & 0.382 \\
\hline Sex, male/female & $99 / 19$ & $28 / 11$ & 0.105 \\
\hline Follow-up, mean $\pm S D$, months & $39.3 \pm 22.39$ & $47.5 \pm 22.03$ & 0.048 \\
\hline Location, L/M/U1 & $30 / 76 / 12$ & $10 / 25 / 4$ & 1 \\
\hline Morphology, elevated/flat or depressed/advanced ${ }^{2}$ & $39 / 69 / 10$ & $24 / 12 / 3$ & 0.005 \\
\hline Tumor size, mean \pm SD, mm & $19 \pm 18.7$ & $22 \pm 17.7$ & 0.36 \\
\hline Histological type, intestinal/diffuse & $93 / 25$ & $37 / 2$ & 0.026 \\
\hline \multicolumn{4}{|c|}{$\begin{array}{l}\text { SD, standard deviation. } \\
1 \text { L, lower part of stomach; M, middle part; U, upper part. } \\
2 \text { Flat or depressed, flat and superficial depressed lesions; elevated, protruding and superficial elevated lesions; advanced, advanced cancers. }\end{array}$} \\
\hline
\end{tabular}

detect malignant lesions at their early stage, but a large prospective study is necessary to investigate an adequate surveillance interval.

The sample size in our study was reasonably large, yet the study has limitations. The major one is the lack of full information on the initial GSN lesions. We retrospectively reviewed previous EGD records of gastric cancer and identified the suspicious preceding lesion. Most lesions had not been recognized at the time of previous EGD and were identified at the review of the records. Thus, the diagnosis of the initial lesion as GSN was based only on endoscopic findings of the EGD records. To increase the diagnostic accuracy, we only enrolled GSNs based on the diagnosis with agreement of all five reviewers. Another issue is the lack of information on the patients' Helicobacter pylori status. Eradication of $H$. pylori largely prevents the development of gastric cancer [16-19] and likely would affect the natural history of GSN [20]. However, in this retrospective study, we were unable to obtain essential data on patients' $H$. pylori status and response to eradication therapy.

In conclusion, despite these limitations, our findings indicate that GSNs are often missed but most usually progress slowly. Even though the rate of progression to cancer is relatively low, rigorous attempts should be made to reduce the miss rate of GSNs at EGD.

\section{Acknowledgments}

Portions of this work were presented at the 23 rd United European Gastroenterology Week in October 2015 in Barcelona and published in abstract form in United Eur Gastroenterol ] 2015; 3 (Suppl): A72-73.

The authors thank Dr. William R. Brown (Denver, Colorado, United States) for assistance in preparation of the manuscript.

\section{Competing interests}

Yuichi Shimodate received a travel grant from United European Gastoroenterology (UEG) to attend UEGW2015, the 23 rd United European Gastroenterology Week, Barcelona, Spain.

\section{References}

[1] Gotoda T, Yamamoto H, Soetikno RM. Endoscopic submucosal dissection of early gastric cancer. J Gastroenterol 2006; 41: 929 -942

[2] Menon S, Trudgill N. How commonly is upper gastrointestinal cancer missed at endoscopy? A meta-analysis Endosc Int Open 2014; 2: E46-50

[3] Voutilainen ME, Juhola MT. Evaluation of the diagnostic accuracy of gastroscopy to detect gastric tumors: clinicopathological features and prognosis of patient with gastric cancer missed on endoscopy. Eur J Gastroenterol Hepatol 2005; 17: 1345-1349

[4] Hosokawa O, Hattori M, Douden K et al. Difference in accuracy between gastroscopy and colonoscopy for detection of cancer. Hepatogastroenterology 2007; 54: 442-444

[5] Japanese GastricCancer Association. Japanese classification of gastric carcinoma: 3rd English edition. Gastric Cancer 2011; 14: 101 - 112

[6] Schlemper RJ, Riddell RH, Kato Y et al. The Vienna classification of gastrointestinal epithelial neoplasia. Gut 2000; 47: 251 - 255

[7] Lauren P. The two histological main types of gastric carcinoma: Diffuse and so-called intestinal-type carcinoma. Acta Pathol Microbiol Scand 1965; 64: $31-49$

[8] Fujita S. Biology of early gastric carcinoma. Pathol Res Pract 1978; 163: $297-309$

[9] Hosokawa O, Kaizaki Y, Nakaya T et al. Retrospective study of endoscopic findings: 250 cases of gastric cancer. Dig Endosc 2000; 12: $136-140$

[10] Rugge M, Cassaro M, Di Mario F et al. The long term outcome of gastric non-invasive neoplasia. Gut 2003; 52: 1111-1116

[11] Park SY, Jeon SW, Jung MK et al. Long-term follow-up study of gastric intraepithelial neoplasias: progression from low-grade dysplasia to invasive carcinoma. Eur J Gastroenterol Hepatol 2008; 20: 966 - 970

[12] Yamada H, Ikegami M, Shimoda T et al. Long-term follow-up study of gastric adenoma/dysplasia. Endoscopy 2004; 36: 390 - 396 
[13] Cho SJ, Cho IJ, Kim CG et al. Risk of high-grade dysplasia or carcinoma in gastric biopsy-proven low-grade dysplasia: an analysis using the Vienna classification. Endoscopy 2011; 43: $465-471$

[14] Jung MK, Jeon SW, Park SY et al. Endoscopic characteristics of gastric adenomas suggesting carcinomatous transformation. Surg Endosc 2008; 22: $2705-2711$

[15] Tsukuma H, Oshima A, Nakahara H et al. Natural history of early gastric cancer: a non-concurrent, long-term, follow up study. Gut 2000; 47: $618-621$

[16] Uemura N, Okamoto S, Yamamoto S et al. Helicobacter pylori infection and the development of gastric cancer. NEJM 2001; 345: 784 789

[17] Fukase K, Kato M, Kikuchi S et al. Effect of eradication of Helicobacter pylori on incidence of metachronous gastric carcinoma after endo- scopic resection of early gastric cancer: an open-label, randomised controlled trial. Lancet 2008; 372: $392-397$

[18] Take S, Mizuno M, Ishiki K et al. The effect of eradicating helicobacter pylori on the development of gastric cancer in patients with peptic ulcer disease. Am J Gastroenterol 2005; 100: 1037 - 1042

[19] Ford AC, Forman D, Hunt RH et al. Helicobacter pylori eradication therapy to prevent gastric cancer in healthy asymptomatic infected individuals: systematic review and meta-analysis of randomised controlled trials. BMJ 2014; 348: g3174

[20] Ito M, Tanaka S, Takata S et al. Morphological changes in human gastric tumours after eradication therapy of Helicobacter pylori in a short-term follow-up. Aliment Pharmacol Ther 2005; 21: 559-566 